\title{
Control of roll and pitch motion during multi-directional balance perturbations
}

\author{
Ursula Margareta Küng • C. G. C. Horlings • \\ F. Honegger · J. E. J. Duysens · J. H. J. Allum
}

Received: 30 April 2008 / Accepted: 10 February 2009 / Published online: 5 March 2009

(C) Springer-Verlag 2009

\begin{abstract}
Does the central nervous system (CNS) independently control roll and pitch movements of the human body during balance corrections? To help provide an answer to this question, we perturbed the balance of 16 young healthy subjects using multi-directional rotations of the support surface. All rotations had pitch and roll components, for which either the roll (DR) or the pitch (DP) component were delayed by $150 \mathrm{~ms}$ or not at all (ND). The outcome measures were the biomechanical responses of the body and surface EMG activity of several muscles. Across all perturbation directions, DR caused equally delayed shifts $(150 \mathrm{~ms})$ in peak lateral centre of mass $(\mathrm{COM})$ velocity. Across directions, DP did not cause equally delayed shifts in anterior-posterior COM velocity. After $300 \mathrm{~ms}$ however, the vector direction of COM velocity was similar to the ND directions. Trunk, arm and knee joint rotations followed this roll compared to pitch pattern, but were different from ND rotation synergies after $300 \mathrm{~ms}$, suggesting an intersegmental compensation for the delay effects. Balance correcting responses of muscles demonstrated both roll and
\end{abstract}

U. M. Küng · C. G. C. Horlings · F. Honegger · J. H. J. Allum ( $\varangle)$ Department of ORL, University ORL Clinic,

University Hospital, Petersgraben 4, 4031 Basel, Switzerland

e-mail: jallum@uhbs.ch

U. M. Küng

e-mail: ukueng@uhbs.ch

C. G. C. Horlings

Department of Neurology, Radboud University Medical Centre,

Nijmegen, The Netherlands

J. E. J. Duysens

Department of Rehabilitation,

Radboud University Medical Centre, Nijmegen, The Netherlands pitch directed components regardless of axial alignment. We categorised muscles into three groups: pitch oriented, roll oriented and mixed based on their responses to DR and DP. Lower leg muscles were pitch oriented, trunk muscles were roll oriented, and knee and arm muscles were mixed. The results of this study suggest that roll, but not pitch components, of balance correcting movement strategies and muscle synergies are separately programmed by the CNS. Reliance on differentially activated arm and knee muscles to correct roll perturbations reveals a dependence of the pitch response on that of roll, possibly due to biomechanical constraints, and accounts for the failure of DP to be transmitted equally in time across all limbs segments. Thus it appears the CNS preferentially programs the roll response of the body and then adjusts the pitch response accordingly.

Keywords Balance corrections · Postural control · Muscle responses $\cdot$ CNS motor programs

\section{Introduction}

If balance corrections are differently organised in the roll (medio-lateral) and pitch (anterior-posterior) directions, exploring these differences may provide insights into the mechanisms underlying falls. A major influence on balance corrections is the biomechanical response of the body, which is different in the roll and pitch planes. For a pure pitch perturbation, the trunk moves in pitch only. In contrast, across a range of perturbation directions from pure roll to roll combined with pitch, both pitch and roll motions of the trunk occur (Carpenter et al. 1999; Grüneberg et al. 2005). Thus, if body motion is different depending on the roll and pitch content of the stimulus, then it might be 
expected that the CNS takes this into account when executing balance corrections, possibly by relying more on those muscles which act efficiently in the roll and pitch planes to correct the pitch motion induced by a roll perturbation.

There are two opposing viewpoints on the directional control of balance corrections. One viewpoint asserts that no differences exist between the roll and pitch commands issued by the CNS, rather a common movement strategy and muscle synergy is used regardless of perturbation direction (Henry et al. 1998a, 1998b; Park et al. 2004; Jones et al. 2008). According to this viewpoint, differences in movement responses or joint torques with perturbation direction can be explained by a simple directional re-weighting of the muscle responses along the body, according to the alignment of lines of muscle action with perturbation directions. It was suggested that this re-weighting would take into account the inherent differences in skeletal geometry that lead to different initial responses of the body to the perturbation in the pitch and roll directions. In contrast, the very fact that the timing of trunk velocity is very different in the roll and pitch planes following multi-directional perturbations to stance, led others to believe that there were too many factors to be taken into account for a single directionally re-weighted response synergy to work effectively (Allum et al. 2003; Carpenter et al. 1999, 2001). Some of the factors influencing differences in roll and pitch balance correcting strategies are the differences in the arrival of roll and pitch stimulus-related sensory information used to generate these strategies (Allum et al. 2008), the directional sensitivity of muscle responses (Carpenter et al. 1999) and the need for different knee flexing strategies in the response to roll and pitch tilts (Allum et al. 2008; Oude-Nijhuis et al. 2007).

Thus another viewpoint that has been developed is that the CNS controls roll and pitch joint torques separately. This idea is not new. Winter et al. (1996) suggested separate control of roll and pitch torques during quiet stance and others argued that this is the case for balance corrections (Allum et al. 2008; Carpenter et al. 2001; Matjacic et al. 2001). Matjacic et al. (2001) argued that control in the medio-lateral and anterior-posterior (AP) directions is decoupled based on the observation that net joint torques in pitch only and the roll only directions were identical to those elicited for combined pitch and roll perturbations of the same magnitude. It could however be argued that this does not implicate different control in the two planes and may provide support for the viewpoint that a common torque strategy is utilized regardless of perturbation direction (Henry et al. 1998a, b). Recent studies in the cat, however, also support the concept of separate roll and pitch muscle synergies. Ting et al examined muscles activity in response to several directions of support surface translation and came to the conclusion that, despite the complex num- ber of muscle patterns involved, these could be resolved into four patterns - two for the lateral directions (left and right) and two for AP directions (backwards-forwards) (Ting and Macpherson 2004; Torres-Oviedo et al. 2006). With three different synergies required (those for lateral perturbations would be similar for the left and right directions, just opposite in polarity), two have been aligned in opposite directions in the pitch plane (equivalent to differences in the toe-up and toe-down synergies in humans (Allum et al. 2003, 2008), it can be expected that the resultant balance correcting joint torques would have different patterns in the roll and pitch planes too. This would lead to different movement strategies for pitch and roll as concluded on the basis of studies on humans (Carpenter et al. 2001; Grüneberg et al. 2005; Matjacic et al. 2001; Winter et al. 1996).

One way to explore the differences in CNS action for the roll and pitch planes is to delay either the pitch or roll component of the stimulus and compare the response to that with no delay. If the roll and pitch correcting commands are organised separately, a delay in one command should not affect the other. Grüneberg et al. (2005) used only delayed roll (DR) tilt stimuli with respect to pitch, in order to focus on the different CNS response organization for these two planes. One of the roll stimulus delay times chosen, that with $150 \mathrm{~ms}$ delay, was designed to shift the earlier roll trunk motion to the time when trunk pitch motion normally occurs if there is no delay (ND) between roll and pitch components of the stimulus. In this way, both roll and pitch commands were forced to act at the same time. This approach worked well in that Grüneberg et al. (2005) were able to show that shifting the roll stimulus merely shifted, but did not alter the roll dependent amplitude characteristics of trunk motion or trunk muscle responses. Their results supported the idea that pitch motion is mainly controlled by the ankle muscles and roll motion by the hip and trunk muscles (Carpenter et al. 2001; Matjacic et al. 2001; Winter et al. 1996), but left a number of important issues unexplored. Most importantly, they did not explore the effect of delaying the pitch component of the tilts in different directions. The lack of an interaction with pitch movements for DR stimuli might not be true for delayed pitch (DP) stimuli. Second, Grüneberg et al. (2005) did not explore the effect of the delays on the primary controlled variable, centre of mass (COM) movement. Third, they did not explore knee and arm (shoulder joint) motion. At these joints, an interaction between roll and pitch corrections could be expected (Allum et al. 2008, Bakker et al. 2006) in addition to any at the trunk. A study of arm and knee joint motion as well as trunk angular motion would seem crucial as these variables show high correlations to COM motion when instability is present (Küng et al. 2009). To explore these issues experiments with both DR and DP components 
to tilt stimuli are required with measurements of shoulder and knee joint motion and muscle activity.

Thus the aim of this study was to provide supporting evidence for separate neural control of roll and pitch body motion during balance corrections. For this purpose, we investigated the balance corrections following support surface tilts with DR and DP stimulus components. One hypothesis we explored was that the biomechanical reactions of the human body in the roll and pitch planes are decoupled from one another and for this reason, the CNS controls motion in these planes independently (Grüneberg et al. 2005). We assumed that this could be revealed using delays in the roll and pitch components of tilt stimuli. An alternative hypothesis would be that one command is simply reweighted by the CNS dependent on direction of body motion (Henry et al. 1998a, b; Jones et al. 2008). Neither hypothesis fit our results, because of the interactions between pitch and roll responses in trunk motion, as well as knee and arm responses, following tilt stimuli with roll components.

\section{Materials and methods}

\section{Subjects}

A total of 16 young healthy subjects without neurologic or orthopaedic deficits were selected [mean age $27 \pm 1$ (SEM) years; height $175 \pm 2.1$ (SEM) $\mathrm{cm}$; and weight $69 \pm 1.8$ (SEM) kg]. All subjects gave witnessed informed, written consent to participate in the experiments according to the Declaration of Helsinki. The Institutional Ethical Review Board of Basel University Hospital approved the study.

\section{Protocol}

The subjects' feet were lightly strapped across the insteps with backward foot movement blocked by heel guides fixed to the upper surface of a movable platform capable of rotating in the pitch and roll directions. The heel guides were adjusted to ensure that the ankle joint axes were aligned with the pitch axis of the platform. The foot straps prevented stepping reactions when stimuli causing stance perturbations occurred. The roll axis had the same height as the pitch axis and passed between the feet. The stance width was standardized $(14 \mathrm{~cm})$ and two handrails of adjustable height were located $40 \mathrm{~cm}$ from the sides of the platform centre. Subjects were informed that they were allowed to grasp the handrails if they needed support. One assistant was presented to lend support in case of a fall, but no falls, or near falls (defined as a need to grasp the handrail or receive assistance) occurred.

Stimuli consisted of rotations of the platform in eight different directions with a constant velocity of $60 \%$ and a constant amplitude of $7.5^{\circ}$. Pitch and roll rotations of the platform were combined to reach the following resulting tilt directions defined in laboratory coordinates (see schema in Fig. 3): forward right $\left(23^{\circ}, 68^{\circ}\right)$, backward right $\left(113^{\circ}\right.$, $\left.158^{\circ}\right)$, backward left $\left(203^{\circ}, 248^{\circ}\right)$, and forward left $\left(293^{\circ}\right.$, $338^{\circ}$ ). We chose those stimulus directions for two reasons. First, each direction would have a pitch or roll component that could be delayed. Pure roll or pure pitch directions would not have both components, and second, to have comparable directions to those used by Grüneberg et al. For all stimulus directions, either the roll or the pitch component of the stimulus could be delayed by $150 \mathrm{~ms}$ (DP or DR) or both components could occur simultaneously with ND. Each perturbation was presented in a random order eight times to the subject. To minimize fatigue, participants were given a $3-4$ min seated rest after the 36th, 73th, 108th and 144th trial. Each trial was preceded by a random $5-15 \mathrm{~s}$ interstimulus delay, which was initiated automatically. During this time period, visual feedback of the subjects' own AP and medio-lateral ankle torque was presented to the subject on a cross with light emitting diodes. This visual feedback was used to standardize prestimulus subject position across trials. Subjects were required to maintain AP ankle torque within a range of $\pm 5 \mathrm{Nm}$ and medio-lateral torque within $\pm 10 \mathrm{Nm}$ of their preferred stance reference values. In response to each perturbation, subjects were instructed to recover their balance as quickly as possible. The visual feedback was switched off at stimulus onset for $5 \mathrm{~s}$.

\section{Data collection}

Recordings of biomechanical and EMG data commenced $100 \mathrm{~ms}$ prior to perturbation onset and were collected for $1 \mathrm{~s}$. To record EMG activity, pairs of silver-silver chloride electrodes were placed approximately $3 \mathrm{~cm}$ apart along the muscle bellies of left tibialis anterior, left soleus, left peroneus longus, left rectus femoris, left biceps femoris, left gluteus medius, left medial deltoid (pars acromialis) and bilaterally on paraspinals at the L1-L2 level of the spine. EMG recordings were analogue band-pass filtered between 60 and $600 \mathrm{~Hz}$, full-wave rectified, and low-pass filtered at $100 \mathrm{~Hz}$ prior to sampling at $1 \mathrm{kHz}$.

Full body kinematics were collected using a threedimensional optical tracking system with 21 infrared emitting diodes (IREDs) (Optotrak, Northern Digital). The Optotrak cameras sampled the IRED signals at $64 \mathrm{~Hz}$ and were placed approximately $4 \mathrm{~m}$ in front of the subject. IREDs were placed bilaterally on the following anatomical landmarks: frontally at the lateral malleolus; center of the patella; frontally at the greater trochanter; anterior superior iliac spine; radial styloid process; elbow axis; acromion; chin; angulus sterni; and on a headband placed just above 
the ears. Three IREDs were placed at the front corners and the left side of the platform to define the pitch and roll movements of the platform. Subjects wore tight fitting shorts and vests to prevent marker movements.

Support surface reaction forces of both feet were measured from strain gauges embedded within the rotating support surface. The strain gauges were located under the corners of the plate supporting each foot. From forces recorded perpendicular to the support surface by the strain gauges under the left foot and the distances to the centre of ankle joint rotation, the AP and lateral ankle torques were calculated for the left foot. Because a difference in strain gauge measures was used for torque calculations, an influence of the platform mass on the torque measures was negligible. A similar system measured forces and torques applied by the right foot. The torques from the left and right foot were added together and displayed to the subject as described above.

\section{Data analysis}

Primary variables of interest were COM displacement and velocity, trunk angular velocity, shoulder and knee joint angular velocity profiles as well as muscle responses of the legs, arms and the trunk.

Following analogue to digital data conversion, biomechanical and EMG signals were averaged offline across each perturbation direction. Zero latency was defined as the onset of platform rotation. Subject averages were pooled to produce population averages for a single direction. The first trial was excluded from data analysis to reduce habituation effects entering the data (Keshner et al. 1987).

\section{Kinematic analysis}

Marker position data from the Optotrak system were digitally filtered at $16 \mathrm{~Hz}$ using a zero phase shift 4th order butterworth filter. Total body COM displacement and velocity were calculated separately for the AP, lateral and vertical directions using a 12 body segment adaptation (Visser et al. 2008) of a 14 segment model (Winter et al. 2003). Two trunk segments (upper and lower trunk) were used instead of four. In addition, we calculated the following angular displacements: absolute upper trunk angle (roll and pitch), pelvis, and head angle, ankle and knee joint angles. Absolute rotation angles of the planes defined by pelvis trunk, and head body segments and the platform surface were calculated using three or four markers to define a plane on these segments. The rotation of this plane was calculated yielding an estimate of the segment rotation. Knee and ankle joint angles were calculated using the angle between the body segments either side of the joint. Arm abduction and rotation were calculated as the angle between the upper arm and upper trunk segments (for further details see Bakker et al. 2006; Visser et al. 2008). Stimulus induced changes were calculated with respect to a pretrigger time interval of $90 \mathrm{~ms}$ ending $10 \mathrm{~ms}$ prior stimulus onset. We concentrated our analysis of body segment motion to that of the upper trunk, the arm angles with respect to the trunk and knee flexion as these motions had been shown to have the strongest correlation to COM linear velocity, following tilt of the support surface (Küng et al. 2009). Peak velocity amplitudes and times for these variables were measured in both population and individual average traces.

\section{EMG analysis}

Each EMG response was corrected for background activity by subtracting the average level of prestimulus activity measured over a $90 \mathrm{~ms}$ period ending $10 \mathrm{~ms}$ prior to perturbation onset. Then techniques similar to those previously employed (Grin et al. 2007; Grüneberg et al. 2005) to determine the response areas of balance correcting responses were used for analysis. Basically response areas were defined over intervals from the onset of balance correcting muscle activity until $150 \mathrm{~ms}$ later. We considered only the first $150 \mathrm{~ms}$ because due to the delay interval of $150 \mathrm{~ms}$ of the DP or DR stimuli, earlier short and medium latency activity in the delayed stimulus responses would also have contributed to measured ongoing balance correcting activity. The onset of the balance correcting responses was defined from the population response for the muscle based on the direction showing the greatest peak activity. From the time of peak activity, the analysis algorithm looked backwards in time to locate the moment when the activity was last below the threshold given by the sum of the mean (set to zero after correction for background activity) plus 2.5 times the standard deviations of the prestimulus activity. Starting at this onset, areas were calculated over an interval of $150 \mathrm{~ms}$ for each individual response. As seen in Fig. 7, this interval contains the primary burst of balance correcting activity.

\section{Torque analysis}

Anterior-posterior torque was calculated over an interval from 140 to $290 \mathrm{~ms}$ post stimulus onset, when the greatest changes are observed (Carpenter et al. 1999). Torque changes were calculated for left and right feet separately and summed to yield for total AP ankle torque.

\section{Statistics}

Our primary analysis concentrated on between-conditions comparisons of ND, DP and DR responses using a repeated measures ANOVA model (condition $\times$ direction). Significant 
main effects $(p<0.05)$ were further explored using one-way ANOVA and post hoc $t$ test comparisons with a Bonferroni correction to account for the effect of comparing three conditions at once.

\section{Results}

An examination of COM velocity in Fig. 1 suggests that the stimulus delays of the platform motion were replicated in all body links with an equal delay for roll, but not for pitch. As we will show, the shift in the roll responses could be observed in balance corrections of all recorded body segments, but not in pitch responses. In this respect, it is possible to describe the roll responses as decoupled from those of pitch. To highlight the directional differences of roll and pitch responses, in support of separate neural controls for these directions of motion, our description of the results has been divided into four sections. First, we present a global picture by considering COM linear and trunk angular motion with respect to the three delay conditions. Second, results for knee and arm joint motions are described in order to reveal whether differences in roll and pitch coupling of motion occurring at these joints match those at the trunk. We concentrated on trunk angular motion as well as knee and arm joint motion, because we had identified in previous studies that these motions had the greatest effect on COM motion (Küng et al. 2009; Oude-Nijhuis et al. 2007). Third, we examined the roll and pitch components of ankle torque across directions and stimulus delay conditions in order to determine if coupling between roll and pitch responses was present in ankle torque. Finally, we analysed muscle activity at various joints with the aim of correlating this activity to segment motion in the roll and pitch directions and thereby establish a neural correlate as evidence for separate controllers in biomechanical responses.

Biomechanical responses of the COM: comparisons with trunk, knee and arm motion

\section{COM motion: timing and vector directions}

Figure 1 shows examples of COM and trunk motion for two stimulus directions. One direction is $158^{\circ}$ (lower graphs of Fig. 1a-d), a pitch tilt almost purely backwards (toe-up) and the other direction is $113^{\circ}$ (upper graphs of Fig. 1a-d), a roll tilt almost purely right. Directions are illustrated by centre schema in Fig. 3. In Fig. 1, the lateral movement of the COM and the roll motion of the trunk were clearly shifted $150 \mathrm{~ms}$ with the DR stimulus, for both stimulus directions (Fig. 1a, c). For the two directions shown (and for all other directions), the peak in lateral COM was at $170 \mathrm{~ms}$ for ND stimuli. This compared to a later peak in $\mathrm{AP} \mathrm{COM}$ at $300 \mathrm{~ms}$ for directions $158^{\circ}$ (Fig. 1b) and $203^{\circ}$ for ND stimuli. Thus the rationale for the $150 \mathrm{~ms}$ delay time, forcing roll and pitch trunk peak velocity to occur simultaneously was achieved. For other
Fig. 1 Average velocity plots for COM velocity (a and b), and trunk velocity (c and d). Lateral and roll plots on the left, anterior-posterior and pitch plots on the right. Plots for two directions of platform tilt are shown $113^{\circ}$ (right and slightly backwards) and $158^{\circ}$ (toe-up and slightly right). Each plot for each of the three delay conditions is the average of eight responses from 15 subjects (120 responses). Stimulus onset is marked by a dotted vertical line. The times of peak velocity of each curve for the no delay (ND) condition is marked by a solid vertical line
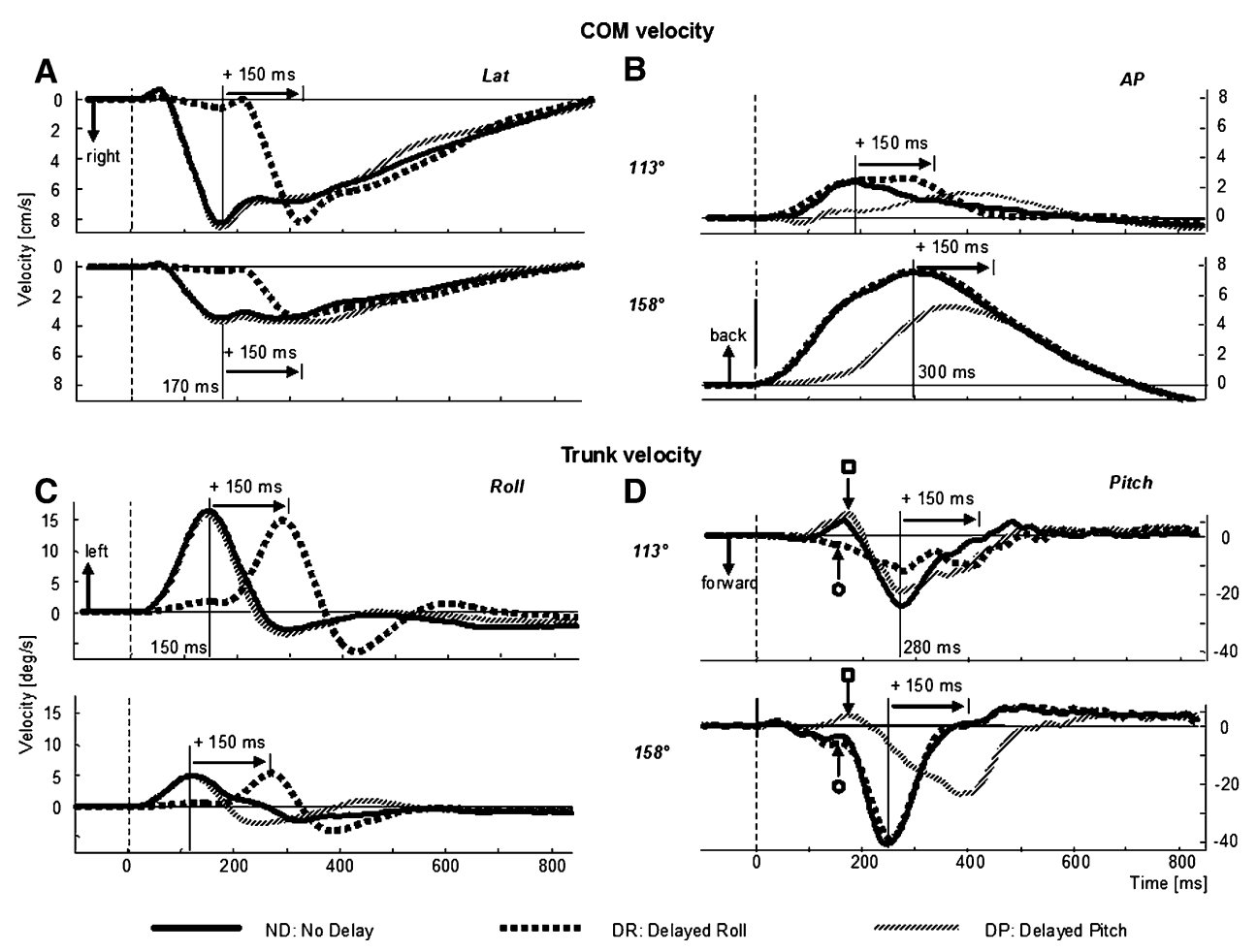


\section{Time to peaks}

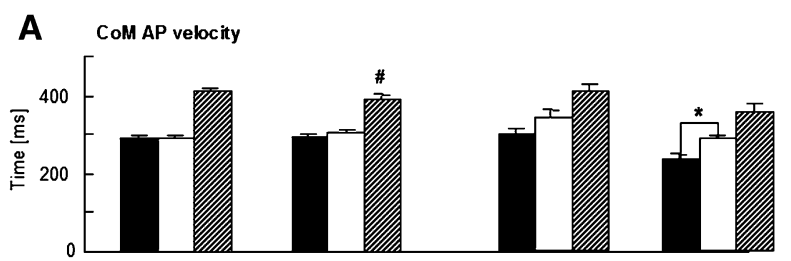

B Trunk pitch velocity

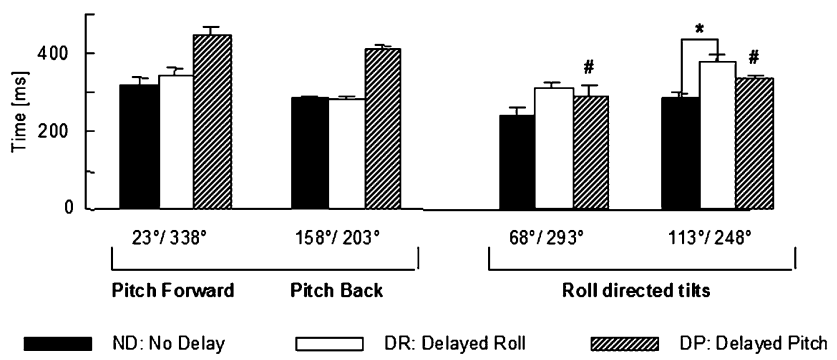

* DR significantly delayed compared to ND

\# DP significantly less than $150 \mathrm{~ms}$ delayed compared to ND

Fig. 2 Times of peak COM AP velocity and trunk pitch velocity across directions for the three delay conditions. The height of each column represents the mean population value based on each subject's mean response (average of eight responses) per direction and the vertical bars standard errors of the mean (SEM). Responses for directions with the same pitch stimulus component, but oppositely directed roll (e.g. $23^{\circ}$ and $338^{\circ}$ ) have been pooled

directions, the peak in AP COM was at different times, compared to the time for near pitch $\left(158^{\circ} / 203^{\circ}\right)$ stimuli and was differently shifted with direction under the delay condition. For example, as shown in Fig. 1b, for the $113^{\circ}$ direction of tilt, the peak in AP COM for DR was shifted almost $150 \mathrm{~ms}$ (rather than having no shift as seen for $158^{\circ}$ in Fig. 1b) compared to ND stimuli. Differences in timing shifts across directions observed in Fig. 1 for AP COM velocities are quantified in Fig. 2. The time of the AP COM velocity was shifted for DP with respect to ND. For the more pitch directed tilts $\left(23^{\circ} / 338^{\circ}, 158^{\circ} / 203^{\circ}\right)$, no shift occurred in AP COM velocity peak for DR stimuli as expected (Fig. 2a). However, peaks in AP COM velocity were shifted for DR with respect to ND stimuli for the more laterally directed backward tilts $\left(113^{\circ}, 248^{\circ}\right)$. These changes in AP COM motion with roll stimulus delay indicate an interaction between roll and pitch responses dependent on stimulus tilt direction.

Despite these changes in timing there were few changes in early vector directions of COM velocities. The polar plots of Fig. $3 \mathrm{a} / \mathrm{b}$ indicate the direction of the COM motion at the two time points, 170 and $300 \mathrm{~ms}$, when these COM velocities have peaks in lateral and AP directions for the ND stimuli in stimulus directions $113^{\circ}$ and $158^{\circ}$, respectively. If the COM motion is independently controlled in roll and pitch, then first, the delay of the roll component of the stimulus (DR) by $150 \mathrm{~ms}$ should cause the COM motion to be pitch oriented at $170 \mathrm{~ms}$ (seen in Fig. 3a). Second, if the pitch component is delayed (DP) $150 \mathrm{~ms}$, then motion should be laterally oriented at $170 \mathrm{~ms}$ (Fig. 3a). However at $300 \mathrm{~ms}$, when a shift in the vector orientation of COM might have been expected with delayed stimulus components, based on the earlier changes in COM velocity at $170 \mathrm{~ms}$ with stimulus delay, only slight differences in the vector orientation of COM velocity between DP, ND and DR stimuli were observed (Fig. 3b). As this result indicates a compensation for earlier changes in COM velocity, we examined whether AP COM position at $800 \mathrm{~ms}$ was altered with stimuli delay. No change was found $(P=0.940)$. However for the DR stimuli, lateral COM position deviated downhill marginally less than for ND stimuli $(\mathrm{P}=0.042)$. These results suggest that delaying roll and pitch components of the stimuli had no overall effect on control of the COM velocity after $300 \mathrm{~ms}$, despite the presence of interaction effects between roll and pitch prior to $300 \mathrm{~ms}$.

\section{Trunk motion: timing and vector directions}

Interactions between pitch and roll responses emerged before and after $300 \mathrm{~ms}$ for angular motion of the upper trunk, the knee and shoulder joints compared to the linear motion of the COM. At approximately $150 \mathrm{~ms}$, when trunk roll velocity peaked for ND stimuli, DP stimuli revealed that roll component of the stimulus caused backward directed pitch motion of the trunk (marked open square in Fig. 1d). This motion was smaller for the backwards DP perturbations, $158^{\circ}$ and $203^{\circ}$ with small roll components (Fig. 1d). The pitch responses revealed with DR stimuli also caused trunk motion with a pitch component at $150 \mathrm{~ms}$ (marked open circle in Fig. 1d), which was, however opposite in direction to that revealed by DP stimuli (see Fig. 1d, see traces marked open circle and open square). Moreover, the pitch trunk velocity for roll directed DR stimuli (for example $113^{\circ}$ DR traces seen in Fig. 1d), appeared to have two peak values, one due to the pitch component of the stimulus at $280 \mathrm{~ms}$ (revealed by DR), the other $150 \mathrm{~ms}$ later due to pitch induced by the DR stimulus. This interaction in pitch responses was not seen for tilts in the two backward pitch directions (Fig. 1d, $158^{\circ}$ ).

In contrast to COM, the vector directions of trunk velocities at $280 \mathrm{~ms}$ for DP and DR stimuli were not aligned with those of the ND stimuli, except for the two backward pitch directions $\left(158^{\circ}\right.$ and $\left.203^{\circ}\right)$. For other tilt directions, vector directions of trunk motion were clearly different for DR and DP stimulus at this time point (Fig. 3d). The earlier timing of the peak in trunk pitch under ND conditions, the shift of the peak in comparison to ND for DR conditions and the lack of a $150 \mathrm{~ms}$ shift for DP stimuli were common characteristics of the more roll 
Fig. 3 Vector directions of COM velocities at 170 (a) and $300 \mathrm{~ms}(\mathbf{b})$, and of trunk velocities at 150 (c) and $280 \mathrm{~ms}(\mathbf{d})$ when these velocities peak (see vertical lines in Fig. 1). For each delay condition, the vector direction of velocity computed from anterior to posterior or pitch and lateral or roll velocities for the COM or trunk, respectively, is shown as a polar plot. The directions of the spokes in the polar plot correspond to the directions of tilt indicated in the middle of the figure

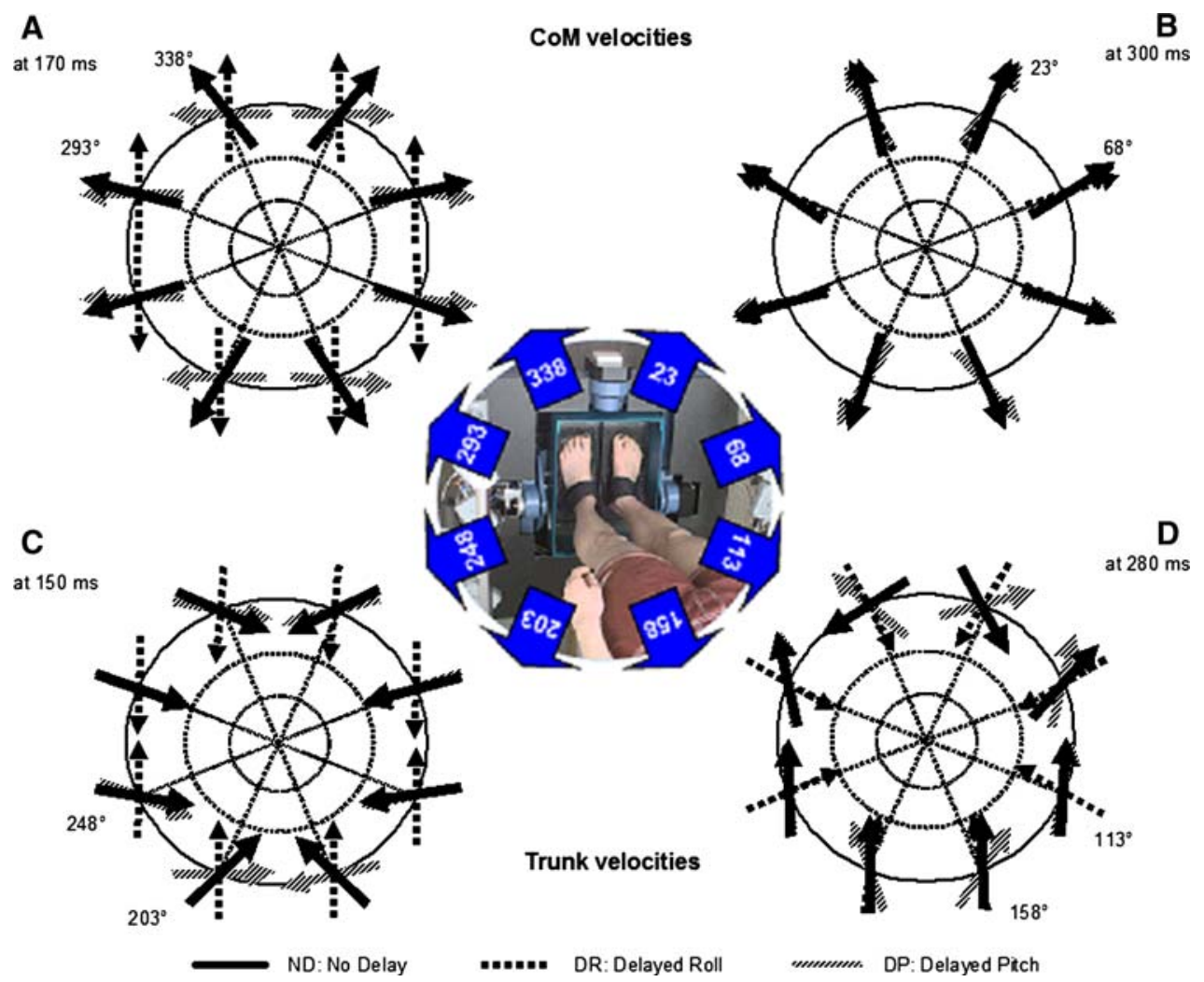

oriented stimuli $\left(68^{\circ} / 293^{\circ}, 113^{\circ} / 248^{\circ}\right.$, see Fig. 2 b). In comparison, the trunk pitch response for more pitch directed stimuli $\left(23^{\circ} / 338^{\circ}, 158^{\circ} / 203^{\circ}\right)$ was clearly shifted for DP and not changed for DR conditions (Fig. 2b). The differences in vector directions for COM at $300 \mathrm{~ms}$ and trunk at $280 \mathrm{~ms}$ suggests that roll components of the stimuli induce pitch motion of the trunk, which is not mirrored in COM motion due to compensation at other body segments, for example, the arms, so that by $300 \mathrm{~ms}$ for COM velocity (and $800 \mathrm{~ms}$ for COM position), no major differences can be observed in COM motion. The question also arises whether this trunk pitch motion is induced directly on the trunk by the tilt perturbations or is induced on the trunk by earlier movements at other body segments, for example, the knees. In the later case, this action would provide evidence of the CNS planning compensatory pitch responses with a roll command.

\section{Amplitudes of COM and trunk angular velocities}

Amplitudes of lateral COM velocity and trunk roll velocity were preserved across delay conditions. It made little difference if the amplitude was examined at the time of the peaks for each subject for each stimulus direction or if the amplitude at the times of the peak in the population average traces was taken (170 and $320 \mathrm{~ms}$ for lateral COM velocity and 150 and $300 \mathrm{~ms}$ for trunk roll velocity for DP and DR stimuli, respectively). There were no differences in the peak amplitudes across stimulus direction [COM: $F(2,84)=0.067 ; P>0.5 ;$ trunk: $F(2,87)=0.021 ; P>0.5]$.

In contrast, delay conditions caused small, but significant changes in the amplitudes of AP COM. A consistently reduced AP COM velocity with DP stimuli occurred for forwards $\left(23^{\circ} / 338^{\circ}\right)$ and backwards $\left(158^{\circ} / 203^{\circ}\right)$ directed stimuli (Fig. 4).

In summary, these results indicate that when the pitch component of the tilt stimulus is delayed, then the timing of the peak velocities of AP COM and trunk pitch are not shifted an amount equal to the delay and amplitudes are not preserved. This effect occurs preferentially for roll directed tilts, that is, those with a larger roll component than pitch. These effects contrast with a lack of effects of stimulus delay on lateral COM and trunk roll responses other than a shift of $150 \mathrm{~ms}$ for DR stimuli. This provides evidence that the roll component of balance correcting responses can be programmed by the CNS independently of the pitch response, but not vice versa. The question arises whether the lack of an effect of the delays on COM motion after $300 \mathrm{~ms}$, despite the clear effect on trunk motion after $300 \mathrm{~ms}$ is due to a compensatory action of the CNS for trunk motion using knee and arm responses.

\section{Knee angular velocities}

In comparison to the trunk, flexion of the knees has the second-most significant influence on lateral and AP motion of 
Fig. 4 Mean amplitudes of COM AP velocity measured from subjects' individual mean response peaks (a). Measurements at $300 \mathrm{~ms}$ (for ND and DR stimuli) and $150 \mathrm{~ms}$ later for DP stimuli, times when population mean has a maximum value (b). The layout of the figure is identical to that of Fig. 2

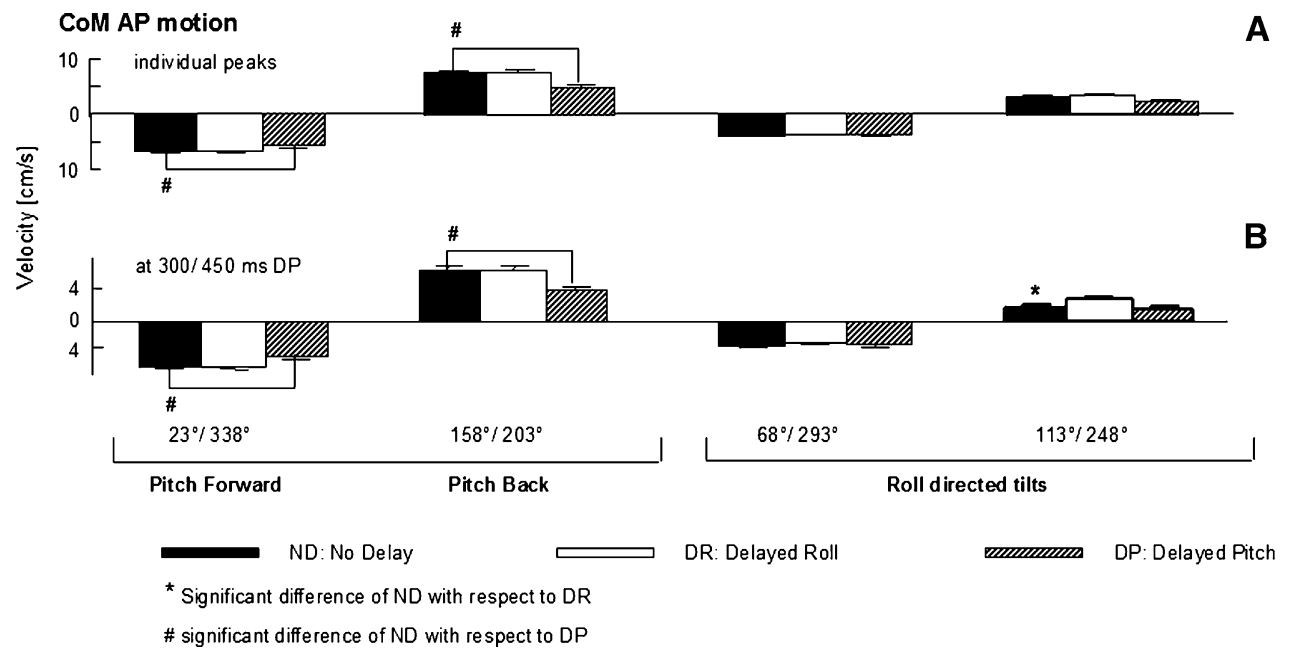

the COM (Küng et al. 2009). The uphill knee flexes and the downhill knee extends (up to approximately $2-3^{\circ}$ to maximum extension) for roll tilts of the support surface (Bakker et al. 2006; Allum et al. 2008). Insufficient flexion and extension leads to an unstable COM position and loss of balance (Küng et al. 2009). Appropriate correction for a roll and/or forward tilt is based on the difference in knee movements. Greater flexion of the uphill knee and extension of the downhill knee provides a greater lateral shift of the COM uphill due to a greater difference between the knee movements. On the other hand, knee flexion will also influence AP COM motion (Oude-Nijhuis et al. 2007). The greater the sum of the two knee flexion movements (zero if one knee flexes an equal amount to the extension of the other), the greater effect on AP COM motion. Thus, we were interested in learning how delaying the roll and pitch components of the stimulus influences knee movements. For this purpose, we examined the difference and sum of knee flexion movements.

Knee flexion movements occurred primarily for forward and/or roll tilts. Knee flexion (both differential and summed) was small for backwards tilts $\left(158^{\circ}\right.$ and $\left.203^{\circ}\right)$. There were two phases in the difference in knee flexionextension velocities with the first relative knee extension having a peak at approximately $130 \mathrm{~ms}$ (preceding the peak in trunk roll velocity, compare Figs. 5a and 1c). This was followed by relative uphill knee flexion, which peaks at approximately $250 \mathrm{~ms}$ (see Table 1 ), as the trunk roll velocity uphill reduced to near zero (compare with trunk roll velocity traces in Fig. 1c). Over perturbation directions, the roll velocity directed profiles of knee flexion appeared to be decoupled from a dependence on the pitch effect of knee flexion, because profile timing was equal for the ND and DP conditions and shifted $150 \mathrm{~ms}$ for the DR condition (see Fig. 5a; Table 1). No changes in the amplitude of differential knee flexion velocity occurred across delay conditions $[F(2,81)=0.224, P>0.05]$.
The sum of the left and right knee velocities divided by two is shown for right and forward tilts of the platform in Fig. 5b. If knee movements were used to predominantly control the pitch rather than the roll displacement of body, then across directions all traces of the sum of left and right knee velocities should be similar to those for the $23^{\circ}$ directed perturbation. For this direction, the response to DR stimuli has the same profile as ND stimuli and the response to DP stimuli is delayed $150 \mathrm{~ms}$ with respect to ND stimuli. For the perturbations with a greater roll than pitch component (see traces for $68^{\circ}$ and $113^{\circ}$ in Fig. 5b), the profile of the sum of the left and right knee velocity was different. The response to DP was shifted earlier and the response to DR was shifted later (Fig. 5b; Table 1). These changes were consistent with changes in the amount of trunk pitch velocity present under ND and DR conditions for near roll perturbations.

\section{Arm angular velocities}

Arm movements also have a strong influence on COM movements (Küng et al. 2009). For lateral tilts, both arms moved laterally downhill, but the amount of abduction and adduction in each arm varies (Küng et al. 2009), hence we considered the difference in arm abduction velocities (see Fig. 6). For roll and backward pitch tilts, both arms rotate forward with most rotation occurring for backwards tilts (Küng et al. 2009), hence we considered the sum of arm rotation velocities.

Arm abduction velocities, considered as the difference between the left and right arms were shifted exactly $150 \mathrm{~ms}$ for DR stimuli across all perturbation directions, with no shifts for DP stimuli (Fig. 6 left). The timing of the peaks in arm abduction velocities was identical to those of trunk velocities, occurring at approximately $150 \mathrm{~ms}$, and prior to the peak in the difference in knee flexion velocities (compare trunk, arm and knee difference traces in Figs. 1c, 5a, 6a; Table 1). 
Fig. 5 Mean population traces of the difference in right and left knee flexion movements (a) leading to a lateral stabilisation of the body and sum of traces of the right and left knee flexion (b) leading to trunk pitch. Responses for the three delay conditions and three directions of right tilt are shown. Knee responses for the direction $158^{\circ}$ are small. The layout of the figure is identical to Fig. 1 with peak responses marked by a solid vertical line

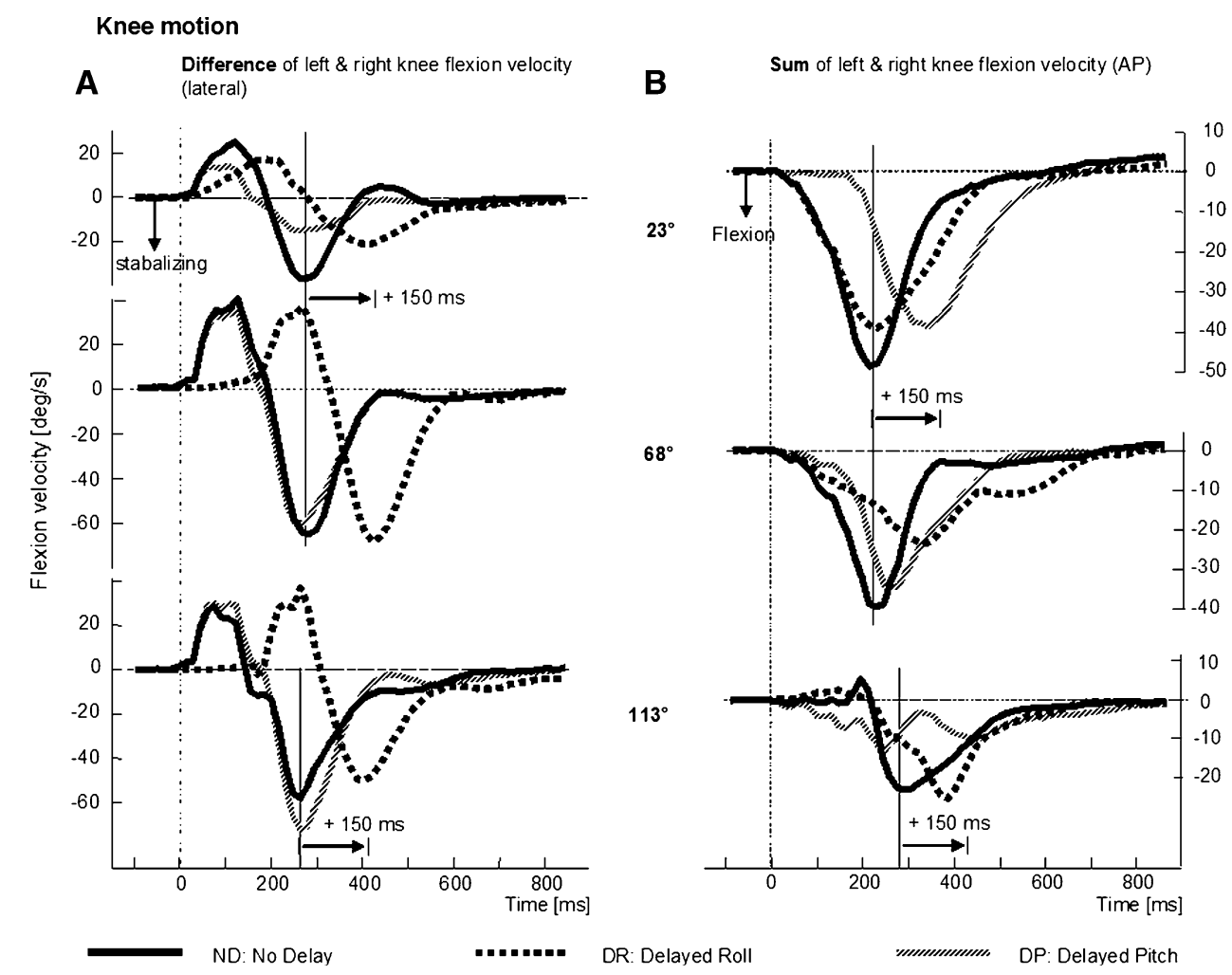

Table 1 Times of peak responses in knee and arm velocities

\begin{tabular}{llll}
\hline \multicolumn{2}{c}{ Knees: differences of left and right, mean $( \pm$ SE $)$} & \\
& $23^{\circ} / 338^{\circ}$ & $68^{\circ} / 293^{\circ}$ & $113^{\circ} / 248^{\circ}$ \\
ND & $277.55( \pm 11.09)$ & $270.51( \pm 12.41)$ & $256.80( \pm 18.85)$ \\
DR & $322.00( \pm 19.28)$ & $416.25( \pm 13.78)$ & $394.68( \pm 16.30)$ \\
DP & $316.56( \pm 22.21)$ & $257.51( \pm 17.44)$ & $251.19( \pm 19.21)$
\end{tabular}

Knees: sum of left and right, mean $( \pm \mathrm{SE})$

$\begin{array}{llll} & 23^{\circ} / 338^{\circ} & 68^{\circ} / 293^{\circ} & 113^{\circ} / 248^{\circ} \\ \text { ND } & 253.13( \pm 26.76) & 231.08( \pm 12.27) & 266.49( \pm 19.92) \\ \text { DR } & 256.62( \pm 16.66) & 318.73( \pm 24.74) & 361.11( \pm 17.14) \\ \text { DP } & 343.11( \pm 25.11) & 27868( \pm 19.11) & 236.30( \pm 17.98)\end{array}$

Arm: sum of the left and right, mean $( \pm \mathrm{SE})$

\begin{tabular}{|c|c|c|c|}
\hline & $68^{\circ} / 293^{\circ}$ & $113^{\circ} / 248^{\circ}$ & $158^{\circ} / 203^{\circ}$ \\
\hline ND & $326.86( \pm 15.27)$ & $295.43( \pm 10.91)$ & $293.81( \pm 6.82)$ \\
\hline DR & $452.01( \pm 14.48)$ & $399.46( \pm 19.57)$ & $323.07( \pm 24.90)$ \\
\hline DP & $320.36( \pm 16.82)$ & $342.03( \pm 14.07)$ & $435.76( \pm 17.27)$ \\
\hline \multicolumn{4}{|c|}{ Arm: difference of the left and right, mean $( \pm S E)$} \\
\hline & $68^{\circ} / 293^{\circ}$ & $113^{\circ} / 248^{\circ}$ & $158^{\circ} / 203^{\circ}$ \\
\hline ND & $145.74( \pm 4.63)$ & $138.78( \pm 2.43)$ & $163.74( \pm 13.93)$ \\
\hline DR & $293.19( \pm 1.86)$ & $292.61( \pm 2.10)$ & $339.05( \pm 29.71)$ \\
\hline DP & $140.51( \pm 1.77)$ & $143.72( \pm 4.25)$ & $153.87( \pm 17.54)$ \\
\hline
\end{tabular}

For arm rotation velocities, a significant difference equal to $150 \mathrm{~ms}$ in the time of peak velocity was observed in the backward pitch directions $\left(158^{\circ} / 203^{\circ}\right)$ between ND and DP stimuli, with no difference for ND and DR stimuli (Fig. 6b).
For near roll stimuli $\left(68^{\circ} / 293^{\circ}\right.$ and $\left.113^{\circ} / 248^{\circ}\right)$, the forward arm rotation did not follow this pattern, but instead matched the pattern seen for AP directed knee motion (compare responses to $68^{\circ}$ and $113^{\circ}$ tilts in Fig. 6). The time of peak arm rotation (rotation as seen in the transverse plane) velocity shifted earlier in time as the perturbation direction was directed more forwards for DP stimuli with no significant difference of the timing being observed for all predominantly roll directed stimuli $\left(68^{\circ} / 293^{\circ}, 113^{\circ} / 248^{\circ}-\right.$ see Table 1). For DR stimuli, this peak shifted progressively later in time as perturbation direction was directed more forward with the difference between DR and ND changing from almost equal for backward stimuli $\left(158^{\circ}\right.$ and $\left.203^{\circ}\right)$ to a delay of $150 \mathrm{~ms}$ for forward and roll stimuli $\left(68^{\circ}\right.$ and $293^{\circ}$ ). In summary, as Table 1 indicates the peak times of arm rotation responses were after those of knee "pitchinducing" flexion responses, possibly indicating that the arm responses were a compensation for the effects of knee flexion on AP COM velocity.

\section{Ankle torques}

Despite the shifts in knee velocity profiles (Fig. 6), both roll and pitch ankle torques (summed for the left and right foot) were delayed consistent with these torques being decoupled from one another. Lateral torque magnitudes at the ankle were of the order of $1 / 20$ of the AP torques. Summed AP torques from both ankles were shifted $150 \mathrm{~ms}$ by DP stimuli, 
Fig. 6 Mean population traces of the difference in right and left arm abduction movements (a), which precede a peak in trunk roll and traces of the sum of right and left forward rotation movements (b), which follow the sum of knee flexion movements. Traces are shown for three delay conditions and three directions of right tilt. Arm responses for the direction $23^{\circ}$ are small. The layout of the figure is similar to that of Figs. 1 and 5

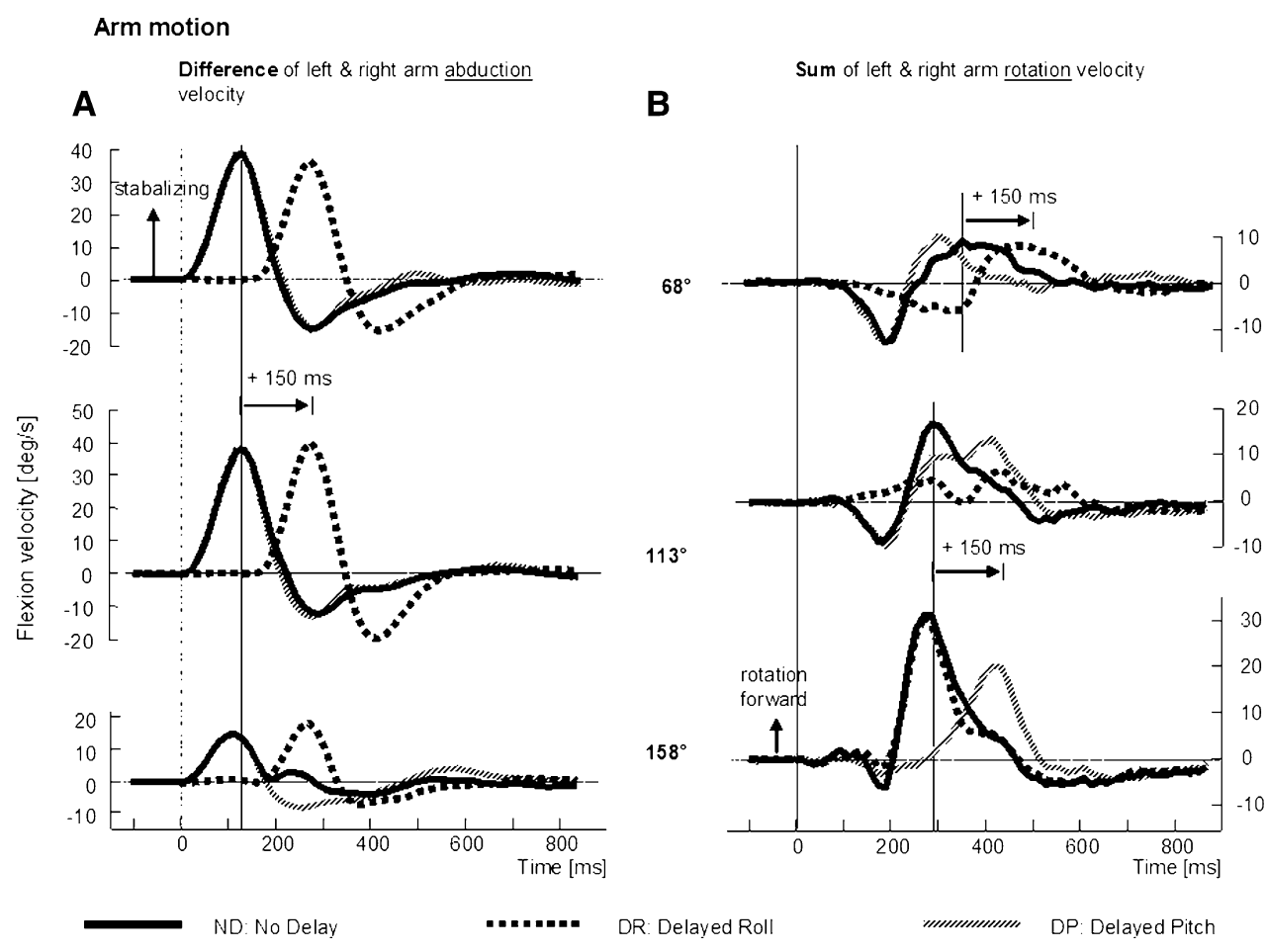

but not at all by DR stimuli. Consider, for example, the AP ankle torque profiles for near roll stimuli $\left(68^{\circ} / 293^{\circ}, 113^{\circ}\right.$ / $248^{\circ}$ ). The uphill and downhill ankle torques were changed by the DR and DP stimuli in one leg, however the torque responses of the contralateral legs had completely opposite polarities. Thus despite these changes at each leg, the effect of the DR stimulus on combined ankle torques was minor even when ankle torque changed rapidly over the balance correcting interval we analysed (140-290 ms). Across all directions combined ankle torque was pitch oriented for ND stimuli and remained so for DR stimuli. The DP stimuli shifted ankle torque profiles $150 \mathrm{~ms}$ with unchanged amplitudes.

\section{EMG responses}

All muscles we examined had responses with varying sensitivities to the pitch and roll components of the stimulus. Figure 7 provides three examples of these differences to delay stimulus for the tilt directions $158^{\circ}$ (backwards) and $248^{\circ}$ (sideways). Tibialis anterior had a pitch sensitivity as its maximal response $\left(158^{\circ}\right.$ in Fig. 7) was significantly shifted $150 \mathrm{~ms}$ for DP stimuli, but not by DR stimuli. Gluteus medius has a roll sensitivity as its maximum response (see traces for $248^{\circ}$ in Fig. 7) was shifted by DR, but not by DP stimuli. However, note that both these muscles were somewhat differently affected by stimulus delay for directions of tilt not eliciting the maximum response amplitude suggesting, for example, tibialis anterior had a roll sensitivity for the $248^{\circ}$ direction and gluteus medius had a pitch sensitivity for the $158^{\circ}$ direction, albeit weak. Responses of an intermediate muscle, rectus femoris with both pitch and roll sensitivity is illustrated by the middle traces of Fig. 7.

To characterise the directional sensitivity of muscle responses, Fig. 8 plots the response amplitudes measured as the area under the curve for the first $150 \mathrm{~ms}$ after response onset for different directions of tilt under the three delay conditions. Each spoke of these polar plots represents the amplitude for a direction of tilt. It is clear from Fig. 8 that tibialis anterior shows little difference in response sensitivity for ND and DR stimuli across directions reinforcing its classification as a pitch directed muscle. Likewise, for gluteus medius, there was little difference between ND and DP stimuli justifying its classification as a roll directed muscle, as supported by the direction of its maximum responsiveness for the ND condition (see arrows on polar plots). Nonetheless, tibialis anterior had a small roll, and gluteus medius had a small pitch sensitivity as indicated by the areas of the polar plots of responses to DP and DR stimuli, respectively, and the arrows indicating the direction of maximum sensitivity under these delay conditions. The polar plot of rectus femoris in Fig. 8 provides an intermediate picture. For this muscle, the area circumscribed by the polar plot is approximately the same size for DP and DR responses, both of which are less than the area of the plot for ND responses.

The area circumscribed in the polar plots, such as those of Fig. 8 was used to categorize the muscle response types as shown in Fig. 9. Thus, pitch sensitivity was defined by the area circumscribed in the polar plot for DR stimuli 
Tibialis anterior left
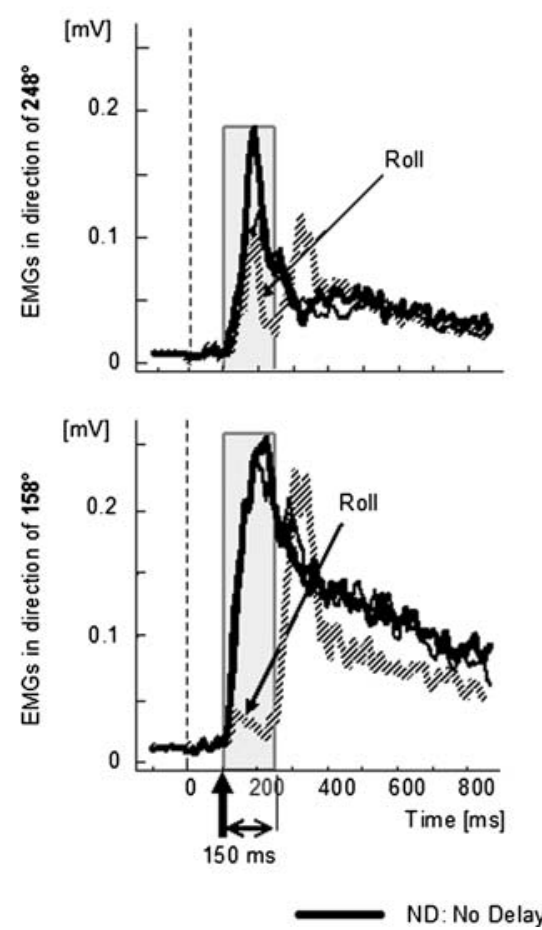

Rectus femoris left
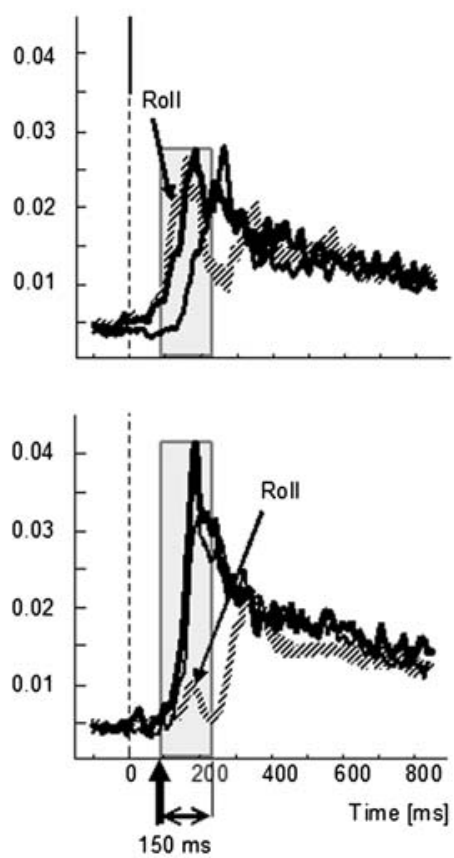

DR: Delayed Roll
Gluteus medius left
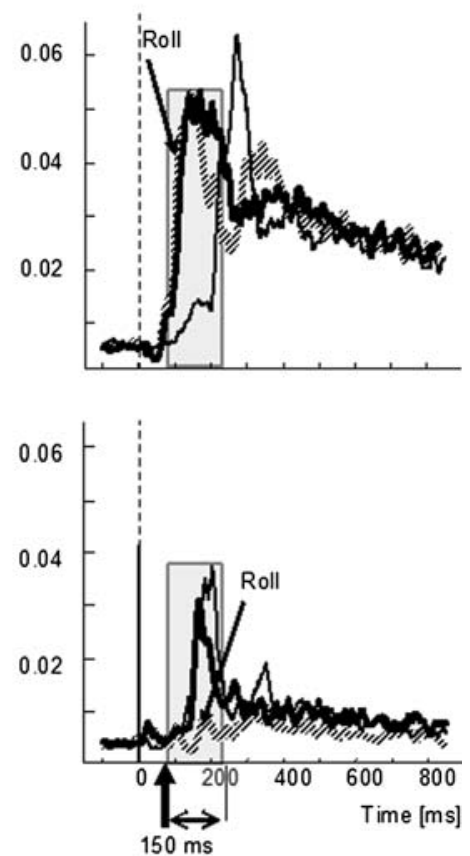

DP: Delayed Pitch
Fig. 7 Mean population traces of EMG activity in tibialis anterior (left set of traces), rectus femoris (middle set of traces) and gluteus medius (right set of traces). The upper row of traces is in response to a tilt in the $248^{\circ}$ direction, the lower set of traces for a $158^{\circ}$ tilt. The onsets of the responses are marked by vertical arrows and the $150 \mathrm{~ms}$ averaging interval by a grey box
Fig. 8 Polar plots of muscle activity over the $150 \mathrm{~ms}$ averaging intervals shown in Fig. 9. The layout of the polar plots is identical to that shown in Fig. 3. Each spoke represents the direction of tilt and radial distance from the plot centre along the spoke the amplitude of the EMG response area according to the scales next to the polar plots. The amplitudes along the spokes are then joined and the direction of the centroid of the figure defines the direction of maximum response sensitivity as indicated by the arrow. Note the different directions of maximum sensitivity for the three stimulus delay conditions for the three muscles

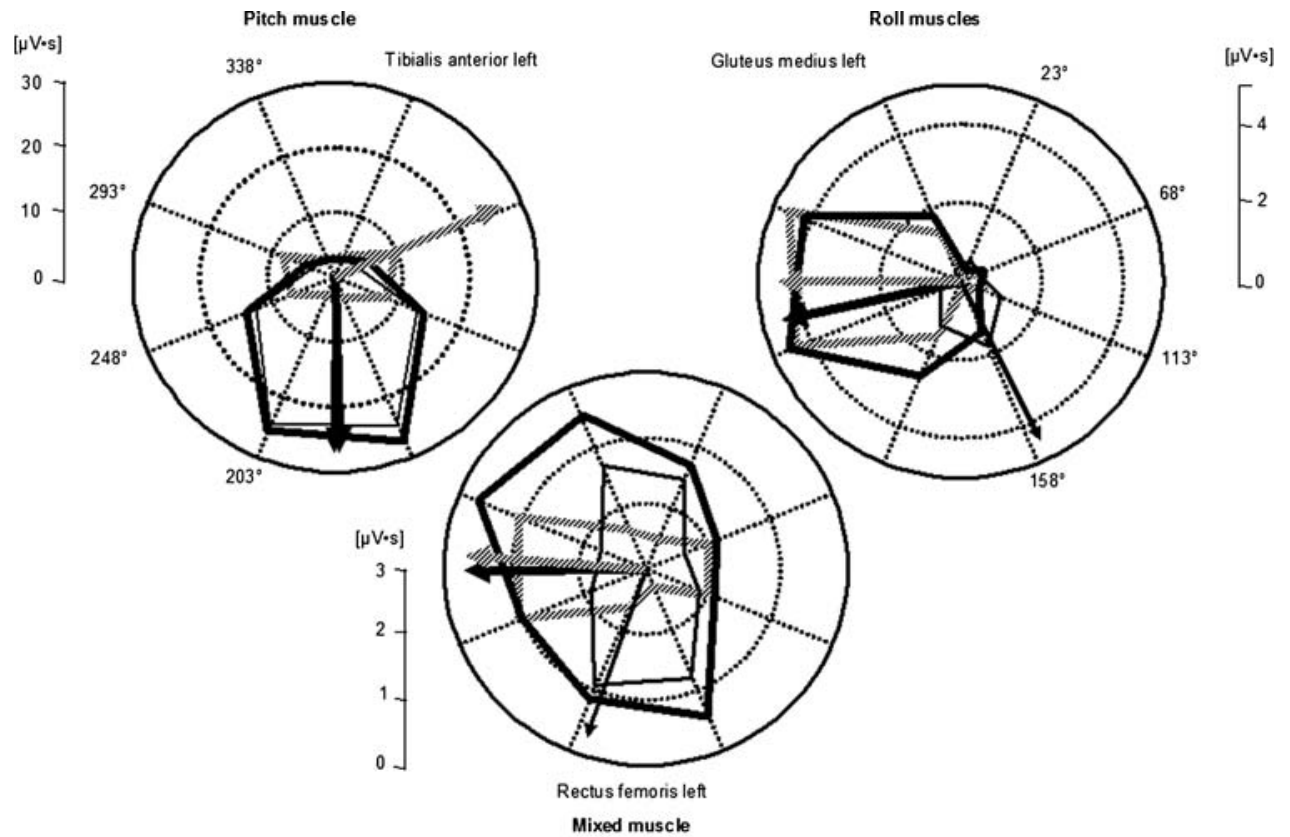

ND: No Delay compared to the area for ND stimuli and that roll sensitivity by DP stimuli, compared to ND stimuli. If pitch sensitivity was significantly greater than the roll sensitivity for a muscle, we termed this as a pitch sensitivity muscle and vice versa, a roll sensitivity muscle. When the two sensitivities had less than a $15 \%$ difference in pitch and roll sensitivity, we termed the muscles as mixed sensitivity muscles. Figure 9 shows how the various muscles we recorded were 


\section{Areas of polar plots}

Pitch muscles
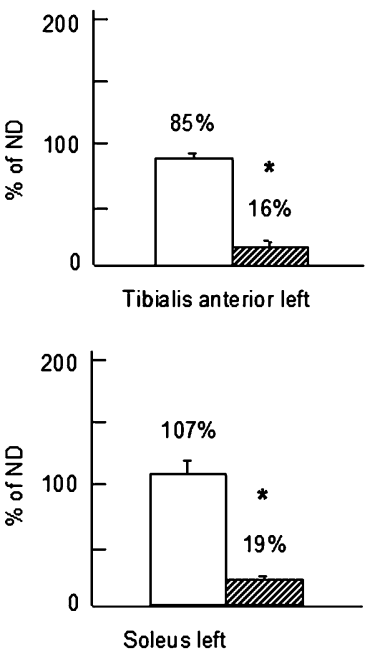
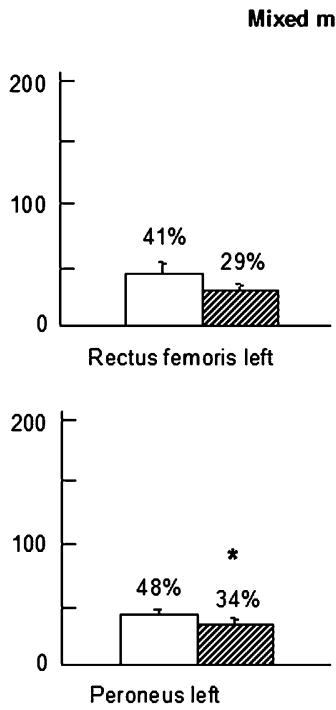
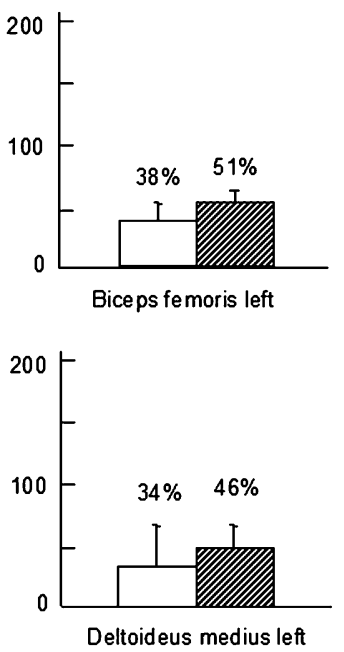

Roll muscles
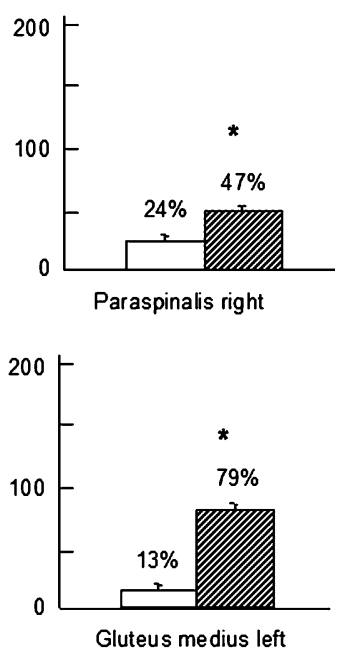

\section{Muscle sensivities \\ Pitch (DR)}

* Pitch significantly different with respect to roll

Fig. 9 Classification of muscle sensitivity based on response areas in the polar plots under the two delay conditions, DR and DP compared to the ND condition (ordinate). Each column represents the mean value for the population and the vertical bar represents the SEM

grouped. The ankle joint muscles, except for peroneus were classified as pitch muscles. The trunk muscles gluteus medius and paraspinals were classified as roll muscles. In contrast, knee (including peroneus) and arm muscles were classified as mixed muscles.

\section{Discussion}

The results of this work add further evidence to the concept that balance corrections in the roll and pitch directions are executed separately by neural command centres. If this command control is managed within the same neural centre or within closely connected, but different neural centres for pitch and roll cannot be determined on the basis of this study. We have been able to extend the work of Grüneberg et al. (2005) for DR stimuli by demonstrating that a delay in the roll component of the stimulus is transmitted faithfully to occur in knee flexion and arm abduction responses at the later time, regardless of the stimulus direction. In the sense that neither the stimulus direction nor the presence or not of a pitch component to the tilt stimulus influenced roll responses, this observation can be interpreted as a control by the CNS of roll decoupled from that of pitch. The findings of Grüneberg et al. (2005), who used different times of DR stimuli, plus earlier work on the biomechanical responses to combined roll and pitch tilts without delays (Allum et al. 2003, 2008; Carpenter et al. 1999), led us to develop the hypothesis that pitch motion would be controlled independently of roll motion too, and that this pitch motion would occur mainly about the ankle and knee joints (Allum et al. 2003; Grüneberg et al. 2005). According to this concept, there would be little interaction between the two forms of control. Our current results as well as recent work in vestibular loss subjects (Allum et al. 2008) indicates that pitch control is dependent on roll, with the dependence increasing as the size of the roll component of the stimulus increases. Thus it appears that CNS is not able to program pitch control independently from roll.

When the pitch component of the stimulus was delayed, trunk, knee and arm movements were not transposed faithfully in time by the amount of the delay. Rather the amount of delay in the response profile and its amplitude depended on the stimulus direction (see Fig. 1, 2, 3, 5, 6). Changes in pitch movements prior to $120 \mathrm{~ms}$ in response to roll directed tilts as shown in right sets of traces in Figs. 1, 5 and 6 are probably due to a purely biomechanical pitch response of the body to roll component of the stimulus. Carpenter et al. (1999) also noted an early pitch response of the trunk following pure roll tilts. An early effect on roll responses in response to the pitch component of the stimulus was not observed in this study and not by Carpenter et al. (1999) for pure pitch tilts. The presence of these early biomechanical changes in pitch for the roll component of the stimulus implies the CNS must take these pitch changes into account when planning the response to the roll perturbation. Most of the later changes in pitch kinematics due to balance corrections could be traced to differences in knee 
flexion movements between the left and right knees across stimulus directions. The flexion of the uphill knee and exclusion of the downhill knee resisted lateral shift of COM downhill by holding the trunk tilted uphill. If not resisted sufficiently, as in, for example, vestibular loss and spinal cerebellar ataxia (SCA) patients with insufficient flexion of the uphill knee and extension of the downhill knee an unstable lateral motion of the COM resulted (Allum et al. 2008; Küng et al. 2009). Because the differential knee action is insufficient in these patients, a reversal of trunk motion downhill occurs. The marked instability in SCA subjects following roll tilts and the inability to program pitch responses to forward tilt adequately (Bakker et al. 2006) would suggest that centres responsible for executing the separate roll and pitch commands, postulated on the basis of the current results, may lie in the vestibulo cerebellum.

It is interesting to speculate whether our results could be explained as an inability of the CNS to generate roll corrections without the use of knee motion, which also induces motion in the pitch plane. It appears that the CNS has other choices in programming roll corrections. For example, when knees flexion was blocked artificially, subjects successfully corrected for roll perturbations using greater than normal arm movements at expense of greater COM motion (Oude-Nijhuis et al. 2008). Thus differential knee flexion may also help control the amount of COM pitch directed motion.

Our analysis of EMG responses under different stimulus delay conditions indicates, as described before, that ankle muscles predominantly control pitch and trunk muscles roll motion of the body (Grüneberg et al. 2005). Our new findings are that arm and knee muscle responses act on both roll and pitch motion of the body as these are equally sensitive to roll and pitch plane components of the stimulus. We assume this action is the primary manner in which the CNS maintains the controlled variable, presumably COM velocity, at a minimum.

The key contribution of knee movements to controlling roll and pitch motion of the body contrasts with the low sensitivity of early passive knee movements to stimulus direction (Allum et al. 2008). This may have the advantage that the knee muscles help control body motion with efferent signals enhancing later proprioceptive feedback in a feedforward manner without early proprioceptive knee responses contributing significantly to the sensory signals initiating and modulating balance corrections. A similar function may be exercised by arm muscles in which stretch reflexes relating to tilt stimuli have not been observed (Allum et al. 2002).

It is interesting to speculate why balance control centres coordinate roll balance corrections, as if these were totally decoupled from those of pitch, but not vice versa. One reason could be simply biomechanical in that roll movement of the trunk occurs earlier than that of pitch following a combined roll and pitch tilt of the support surface (Allum et al. 2002; Carpenter et al. 1999). Furthermore, complete proprioceptive and vestibular information on the stimulus roll characteristics appears to reach the CNS prior to the arrival of pitch directed information (Allum et al. 2008). Thus from timing considerations alone, the CNS may need to carry out the necessary programming and release of the response to roll tilt prior to that for the pitch tilt.

This report presented data on eight directions of tilt each with a roll and pitch component. For completeness, it would have been advisable to have the pure pitch and roll directions as well as $45^{\circ}$ directions with equal components of pitch and roll for the three delay conditions. Data for the pure pitch and roll directions are available in prior publications (Allum et al. 2008; Bakker et al. 2006). Also we have no reason to believe that responses from the directions 45 , 135,225 and 315 in our nomenclature could not be predicted from the current results either side of these directions that is from the current 23, 63, 113, 158, 203, 248, 293 and 338 responses. An expansion of our protocol on the same subjects would probably have been too tiring for them.

These results add further evidence to the differences between the control strategies in centres generating balance correcting responses for bipedal and quadrupedal stance (Allum et al. 2008). Some authors speculated on the similarities based on pitch plane responses (Dunbar et al. 1986; Horak and Macpherson 1996). Roll responses are fundamentally different between bipedal and quadrupedal stance. Firstly, in humans, the motion of the upper trunk is in the opposite direction to that of the pelvis on roll tilt (Allum et al. 2002, 2008). This is a completely different biomechanical response from that of quadrupeds, where trunk and pelvis move in the same direction as the roll tilt of the support surface (Macpherson et al. 2007). The movement of the trunk on the pelvis provides a completely different biomechanical situation in biped stance. In quadrupeds, the uphill knee must flex in order to shift the body laterally uphill, whereas in bipeds the uphill knee must flex in order to hold the trunk in the uphill position compensating for the lateral shift of the pelvis downhill. The current research indicates that the functional pitch plane effect of the knee action in bipeds is presumably not present in quadrupeds due to stance on four legs. We presume that the presence of a different knee action plus simultaneous forelimb action in quadrupeds leads to a different and possibly reduced pitch motion during roll balance control in quadrupeds. Nonetheless, it would be interesting to explore changes in balance corrections for roll tilt when humans are asked to respond to roll tilt in a quadrupedal position in order to determine if the roll responses can be programmed separately from pitch as indicated here. 
In the sagittal plane, increasing use of knee movements was seen, dependent on stimulus velocity (Runge et al. 1999). These results provide a conceptional focus different from the notion that the degrees of freedom are reduced in balance control (Bernstein 1967; Horak and Nashner 1986). While this concept might hold for backward tilts, in the roll plane, the control of the degrees of freedom is quite complex as the knees are controlled independently as are the arms leading to a cross-coupling effect of motion in the pitch plane. The essential question we have tried to address is whether the CNS programs the roll and pitch movement of body independently. The very fact that either the pitch or the roll component of the stimulus could be delayed $150 \mathrm{~ms}$, yet at the completion of the balance correction the overall effect on COM motion was identical to the effect with no delay, would suggest that the CNS programs the roll and pitch motion independently, but that both responses interact in a linear manner biomechanically. Interestingly, when individual segments were examined, it was very apparent that the underlying segment motions in the roll and pitch planes were not independent of one another. Specifically this is very apparent from knee muscle responses, which have almost equal pitch and roll plane sensitivities. The delay of knee flexion movements with respect to trunk roll, and arm movements with respect to the knees, indicates that these movements are not a biomechanical response to the tilt stimulus, rather a compensating balance correction acting to stabilise early trunk motion.

We have assumed here that biomechanical responses prior to $120 \mathrm{~ms}$, interact linearly and that the only effect of the delay was to shift the early biomechanical responses of $150 \mathrm{~ms}$. For our $7.5^{\circ}$ support surface tilts, this appears to be a valid approximation, as segment angle changes are maximally $6^{\circ}$ (Allum et al. 2003). Amplitudes of pitch responses were generally not altered. Roll amplitudes were unchanged with delay conditions. For larger amplitude tilts, this may not be a valid approximation. The amplitude of tilt for which the effect of the delay leads to a fundamentally different response needs to be investigated, possibly with modelling techniques. The lack of such an investigation limits the application of our findings to tilt amplitudes greater than those we investigated.

Although there was a clear interaction between the pitch and roll motion of the trunk, knees and arms induced by tilt of the support surface, delaying either the pitch or roll component did not influence the overall COM velocity response of the body after $300 \mathrm{~ms}$. We had not expected that changes we observed with stimulus delays to pitch would lead to interactions at this level and be compensated in COM responses. Again this reinforces our conclusion that the CNS can program balance corrections in the pitch and roll planes independently of one another, even if interactions exist between the two planes at the level of the arms and knees. Interestingly the form interactions took implied that roll control is programmed first and the pitch control must take into account previously occurring effects on pitch due to roll commands. In this sense, pitch control is not independent of roll. The question arises within this context as whether the roll first action represents a preferred plane of action. Preferred planes of action for head-neck movements has been suggested as a technique to simplify sensory-motor transformations serving motor control and a way to minimize neural operations (Graf et al. 1995).

Acknowledgments This project was supported by a grant from the Swiss National Research Foundation (No. 32000-117950) to J.H.J. Allum.

Conflict of interest statement The authors declare that they have no conflict of interest, financial or otherwise, related to the submitted manuscript or the associated research.

\section{References}

Allum JHJ, Carpenter MG, Honegger F, Adkin AL, Bloem BR (2002) Age-dependent variations in the directional sensitivity of balance corrections and compensatory arm movements in man. J Physiol 542:643-663

Allum JHJ, Carpenter MG, Honegger F (2003) Directional aspects of balance corrections in man. Employing multidirectional perturbations to better understand dynamic postural control in normal and balance-deficient populations. IEEE Eng Med Biol Mag 22:37-47

Allum JHJ, Oude Nijhuis LB, Carpenter MG (2008) Differences in coding provided by proprioceptive and vestibular sensory signals may contribute to lateral instability in vestibular loss subjects. Exp Brain Res 184:391-410

Bakker M, Allum JHJ, Vissser JE, Grüneberg C, van de Warrenburg BPC, Kremer BH, Bloem BR (2006) Postural responses to multidirectional stance perturbations in cerebellar ataxia. Exp Neurol 202:21-35

Bernstein N (1967) The co-ordination and regulation of movements. Pergamon, London

Carpenter MG, Allum JHJ, Honegger F (1999) Directional sensitivity of stretch reflexes and balance corrections for normal subjects in the roll and pitch planes. Exp Brain Res 129:93-113

Carpenter MG, Allum JHJ, Honegger F (2001) Vestibular influences on human postural control in combinations of pitch and roll planes reveal differences in spatiotemporal processing. Exp Brain Res 140:95-111

Dunbar DC, Horak FB, Macpherson JM, Rushmer DS (1986) Neural control of quadrupedal and bipedal stance: implications for the evolution of erect posture. Am J Phys Anthropol 69:93-105

Graf W, de Waele C, Vidal PP, Wang DH, Evinger C (1995) The orientation of the cervical vertebral column in unrestrained awake animals. II. Movement strategies. Brain Behav Evol 45:209-231

Grin L, Frank J, Allum JHJ (2007) The effect of voluntary arm abduction on balance recovery following multidirectional stance perturbations. Exp Brain Res 178:62-78

Grüneberg C, Duysens J, Honegger F, Allum JHJ (2005) Spatio-temporal separation of roll and pitch balance-correcting commands in humans. J Neurophysiol 94:3143-3158

Henry SM, Fung J, Horak FB (1998a) EMG responses to maintain stance during multidirectional surface translations. J Neurophysiol 80:1939-1950 
Henry SM, Fung J, Horak FB (1998b) Control of stance during lateral and anterior/posterior surface translations. IEEE Trans Rehabil Eng 6:32-42

Horak FB, Macpherson JM (1996) Postural orientation and equilibrium. In: Rowell LB, Shepherd JT (eds) Handbook of physiology. Exercise: regulation and integration of multiple systems, sect. 12. Oxford University Press, New York, pp 255-292

Horak FB, Nashner LM (1986) Central programming of postural movements: adaptation to altered support-surface configurations. J Neurophysiol 55:1369-1381

Jones SL, Henry SM, Raasch CL, Hitt JR, Burn JY (2008) Responses to multi-directional surface translations involve redistribution of proximal versus distal strategies to maintain upright posture. Exp Brain Res 187:407-417

Keshner EA, Allum JH, Pfaltz CR (1987) Postural coactivation and adaptation in the sway stabilizing responses of normals and patients with bilateral vestibular deficit. Exp Brain Res 69:77-92

Küng UM, Horlings CGC, Honegger F, Kremer HPH, Bloem BR, van de Warrenburg BPC, Allum JHJ (2009) Postural instability in cerebellar ataxia: correlactions of knee, arm and trunk movements to center of mass velocity. Neuroscience 159:390-404

Macpherson JM, Everaert DG, Stapley PJ, Ting LH (2007) Bilateral vestibular loss in cats leads to active destabilization of balance during pitch and roll rotations of the support surface. J Neurophysiol 97:4357-4367

Matjacic Z, Voigt M, Popovic D, Sinkjaer T (2001) Functional postural responses after perturbations in multiple directions in a standing man: a principle of decoupled control. J Biomech 34:187-196
Oude-Nijhuis L, Bloem BR, Munneke M, Honegger F, Allum JHJ (2007) Incorporating voluntary knee flexion into nonanticipatory balance corrections. J Neurophysiol 98:3047-3057

Oude-Nijhuis L, Hegeman J, Bakker M, Van Meel M, Bloem BR, Allum JHJ (2008) The influence of knee rigidity on balance corrections: a comparison with responses of cerebellar ataxia patients. Exp Brain Res 187:181-191

Park S, Horak FB, Kuo AD (2004) Postural feedback responses scale with biomechanical constraints in human standing. Exp Brain Res 154:417-427

Runge CF, Shupert CL, Horak FB, Zajac FE (1999) Ankle and hip postural strategies defined by joint torques. Gait Posture 10:161-170

Ting LH, Macpherson JM (2004) Ratio of shear to load ground-reaction force may underlie the directional tuning of the automatic postural response to rotation and translation. J Neurophysiol 92:808-823

Torres-Oviedo G, Macpherson JM, Ting LH (2006) Muscle synergy organization is robust across a variety of postural perturbations. J Neurophysiol 96:1530-1546

Visser JE, Allum JHJ, Esselink RA, Speelman JD, Borm GF, Bloem BR (2008) Subthalamic nucleus stimulation and levodopa-resistant postural instability in Parkinson's disease. J Neurol 255:205-210

Winter DA, Prince F, Frank JS, Powell C, Zabjek KF (1996) Unified theory regarding $\mathrm{A} / \mathrm{P}$ and $\mathrm{M} / \mathrm{L}$ balance in quiet stance. J Neurophysiol 75:2334-2343

Winter DA, Patla AE, Ishac M, Gage WH (2003) Motor mechanisms of balance during quiet standing. J Electromyogr Kinesiol 13:4956 